\title{
NANOTECNOLOGIA E NANOBIOTECNOLOGIA: ESTADO DA ARTE, PERSPECTIVAS DE INOVAÇÃO E INVESTIMENTOS
}

\section{NANOTECHNOLOGY AND NANOBIOTECHNOLOGY: STATE OF THE ART, PERSPECTIVES OF INNOVATION AND INVESTMENTS}

\author{
Kleber Vieira Pina ${ }^{1}$; Luciano Rodrigues Pinto ${ }^{2}$; Raquel Barbosa Moratori ${ }^{3}$; Cristina Gomes de \\ Souza $^{4}$; Rafael Garcia Barbastefano ${ }^{5}$ \\ ${ }^{1}$ Centro Federal de Educação Tecnológica - Rio de Janeiro - Brasil - kleber@,fgv.br \\ ${ }^{2}$ Centro Federal de Educação Tecnológica - Rio de Janeiro - Brasil - luciano@yahoo.com.br \\ ${ }^{3}$ Centro Federal de Educação Tecnológica - Rio de Janeiro - Brasil - rmoratori@yahoo.com.br \\ ${ }^{4}$ Centro Federal de Educação Tecnológica - Rio de Janeiro - Brasil - cgsouza@,cefet-rj.br \\ ${ }^{5}$ Centro Federal de Educação Tecnológica - Rio de Janeiro - Brasil - barbastefano@gmail.com.br
}

\begin{abstract}
Resumo
Nanotecnologia consiste na habilidade de manipular a matéria em nanômetros para criar estruturas com uma organização molecular diferenciada. O termo nanobiotecnologia refere-se à interface entre a nanotecnologia com a biologia tendo por objetivo a criação de novos materiais $e$ o desenvolvimento de novos produtos e processos baseados na crescente capacidade da tecnologia moderna de ver e manipular átomos e moléculas. Trata-se de uma área do conhecimento com grande potencial de geração de inovações e que vem despertando interesses econômicos $e$ comerciais ao mesmo tempo em que despontam debates em torno de questões éticas e ambientais. O objetivo do artigo é, a partir do levantamento do estado da arte, procurar traçar um panorama atual e identificar futuras possibilidades de utilização dessa tecnologia. Para a elaboração do trabalho foram realizados estudos na base Web of Science e junto a United States Patent and Trademark Office além do levantamento de investimentos de capital de risco que vêm sendo feitos em nanobiotecnologia e em outras nanotecnologias.
\end{abstract}

Palavras-chave: Nanotecnologia, Nanobiotecnologia, Inovação, Capital de risco.

\section{Introdução}

Muito se tem discutido sobre a tendência de miniaturização que abrange desde computadores e periféricos a máquinas responsáveis pela produção dos mais diversos itens. Entretanto, um aspecto que tem inquietado o meio acadêmico nos últimos anos, relaciona-se a miniaturização de uma forma supreendentemente diferente, não mais se restringindo a miniaturizar 
máquinas e equipamentos mas em fabricá-los em escala atômica, ou melhor dizendo, em escala nanométrica.

O obstáculo de se alcançar a estrutura formadora dos materiais já foi ultrapassado sendo que novos desafios surgem a medida em que o foco, hoje, não encontra-se mais direcionado à simples observação desta estrutura, mas sim, na possibilidade de sua modificação a fim de atender requisitos específicos em determinadas aplicações.

Surgiu assim a nanotecnologia que consiste na habilidade de manipular a matéria em nanômetros para criar estruturas com uma organização molecular diferenciada. Já o termo nanobiotecnologia refere-se à interface entre a nanotecnologia com a biologia tendo por objetivo a criação de novos materiais e o desenvolvimento de novos produtos e processos baseados na crescente capacidade da tecnologia moderna de ver e manipular átomos e moléculas.

No que tange essa nova área do conhecimento verifica-se que ciência e inovações tecnológicas caminham juntas e que fortes laços de reciprocidade refletem-se no crescimento da produção acadêmica e em novas utilizações práticas.

O objetivo do artigo é, a partir do levantamento do estado da arte, procurar traçar um panorama atual e identificar futuras possibilidades de utilização da nanotecnologia e da nanobiotecnologia. Como fontes de pesquisa, foram utilizados livros especializados no assunto, artigos disponíveis nas bases de consulta mantidas pela CAPES, páginas eletrônicas e outras entidades que, mesmo indiretamente, forneceram subsídios para pesquisa, tais como centros de informações sobre patentes e de investimentos. Especificamente foram realizados estudos na base Web of Science e junto a United States Patent and Trademark Office, além do levantamento de investimentos de capital de risco que vêm sendo feitos em nanobiotecnologia e em outras nanotecnologias.

\section{Nanotecnologia e nanobiotecnologia}

Nanotecnologia é o passo posterior a miniaturização e está intimamente ligada a composição física dos produtos. Desde o tempo dos filósofos gregos paira sobre a humanidade a dúvida acerca da possibilidade de se chegar a estrutura formadora dos materiais, ou seja, ao elemento formador universal. A necessidade de se chegar ao "tijolo" fundamental de tudo o que existe ronda a ciência e mobiliza diversas áreas do conhecimento, tais como matemática, química, física e biologia que, unidas, tentam não apenas conhecer em profundidade, mas também, manipular a estrutura básica de todos os materiais identificados pelo homem (SILVA, 2004).

Em essência, a nanotecnologia consiste na habilidade de manipular a matéria em nanômetros para criar estruturas com uma organização molecular diferenciada. Seria algo como montar uma 
molécula do jeito desejado, utilizando átomos como peças fundamentais. Nanômetro (nm) é uma unidade de medida que corresponde à bilionésima $\left(10^{-9}\right)$ parte do metro. É tipicamente o tamanho de uma molécula pequena. Para que se ter uma idéia, os átomos têm dimensões de aproximadamente 0,1 a 0,4 nm, já um vírus tem de 10 a 100 nm (KRUMMENACKER \& LEWIS, 1995).

Pela reduzida escala em que atua esta tecnologia pode-se sintetizar a matéria da forma que for mais adequada à utilização desejada. Modifica-se o arranjo de átomos e moléculas visando-se um produto final mais resistente, mais barato, mais leve, mais preciso, mais puro e mais adequado. Neste sentido, potencialmente falando, a nanotecnologia possui o poder de revolucionar a forma com que se imagina, trata e manuseia a formação de materiais.

Conforme orienta Tsapatsis (2002), com o desenvolvimento da microeletrônica nos defrontamos cada vez mais com estruturas em escalas abaixo de 100nm. A transição da microtecnologia (dimensões laterais de 0,1 a $100 \mathrm{~mm}$ ) para a nanotecnologia (dimensões laterais de 0,1 a $100 \mathrm{~nm}$ ) requer a habilidade de fabricar estruturas menores, assim como a capacidade de exploração e aplicação de fenômenos físicos novos que acontecem em escalas nanométricas.

Dentre os diversos cientistas e estudiosos ligados à nanotecnologia nos tempos modernos pode ser citado Richard Feynman, americano ganhador do Prêmio Nobel de Física, que já na década de 50 anunciava a importância do manuseio dos materiais em nível atômico ao sugerir que, em um futuro não muito distante, toda a enciclopédia britânica poderia ser armazenada na cabeça de um alfinete (FEYNMAN, 1992).

O próprio termo "nanotecnologia" foi introduzido para descrever a visão de Feynman a respeito de fábricas que utilizariam nanomáquinas para manufatura de produtos complexos, incluindo nanomáquinas adicionais, isto é, máquinas que construiriam máquinas auxiliares. Desta forma pode-se afirmar que, de posse da engenharia, este conceito indica uma forte potencialidade para o desenvolvimento de equipamentos com precisão atômica, utilizando materiais superiores e, teoricamente, a custo reduzido.

Conforme Lévy (1988) toda matéria - sólidos, líquidos, gasosos e mesmo seres vivos - é formada por átomos e que, em verdade, tudo o que podemos ver, tocar ou sentir é constituído de um número finito e, bem reduzido, de átomos diferentes. Desta forma o que diferencia uma mesa de um homem, por exemplo, é a organização desses átomos.

Verifica-se, portanto, que qualquer modelo de produção voltado para nanotecnologia se constituirá, basicamente, na arrumação de átomos e moléculas de forma pré-definida. Conforme orientações de Drexler (1986), a tecnologia atual realiza a organização de átomos de forma extremamente bruta, onde, mesmo nos microchips mais avançados, os átomos são organizados em grupos e de forma ainda irregular. 
Já o termo nanobiotecnologia refere-se à interface entre a nanotecnologia com a biologia. Pode significar também qualquer aplicação da nanotecnologia em pesquisa biológica, descoberta de drogas e sua comercialização, ferramentas de diagnóstico, remédios ou novos biomateriais (PAULL et al., 2003). Dentro deste conceito Kotler \& Simon (2004) mencionam a criação de compostos em nível molecular que produzirão materiais inteligentes capazes de "emular sistemas biológicos e usar aprendizagem adaptativa para se auto-reconstrutir".

\section{Estado da arte da pesquisa e desenvolvimento tecnológico}

O estado da arte significa, principalmente, descrever os movimentos da pesquisa científica em determinado momento e que temas estão norteando as principais linhas de estudo. Para efeito do presente trabalho foram utilizadas como fontes de pesquisa as bases de artigos científicos disponíveis na internet, mais precisamente, as bases Web of Science e o Portal de Periódicos da CAPES que abrangem respeitadas revistas e periódicos científicos em âmbito mundial.

Para se comprovar a atualidade do tema junto ao meio acadêmico, foi realizado um estudo quantitativo junto a base do Web of Science, a fim de verificar quanto, em termos numéricos, está sendo produzido e divulgado por revistas e jornais científicos ao longo dos anos sobre nanotecnologia, especificamente. $\mathrm{O}$ resultado do estudo, apresentado na Figura 1, demonstra que o número de artigos a respeito do tema vem aumentando exponencialmente a partir do final da década de 80 .

Figura 1 - Publicações em nanotecnologia catalogadas no Web of Science (1988-2003)

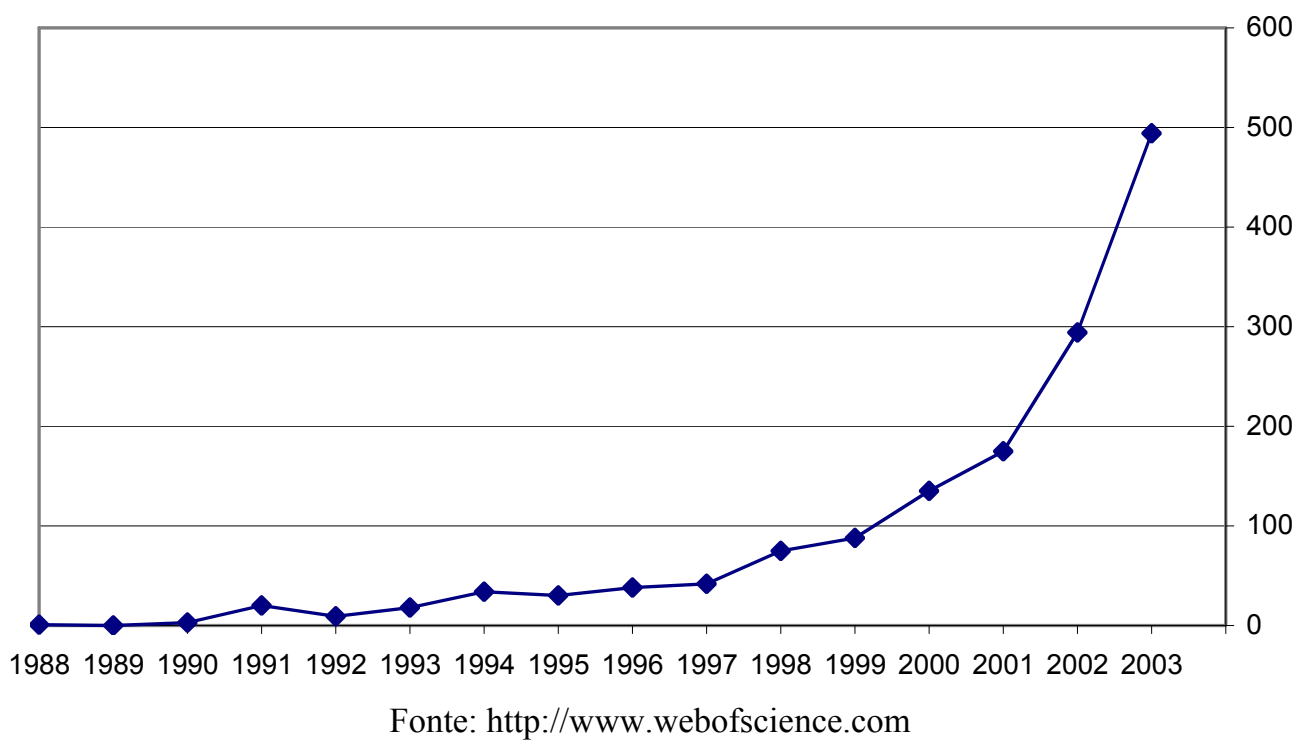

A partir do resultado da pesquisa pode-se inferir, minimamente, que:

- Existe, hoje, um grande interesse acadêmico envolvendo o assunto; 
- Existem, ainda, várias proposições teóricas não respondidas, em sua plenitude, sobre o tema, onde alguns aspectos interessantes dentro deste contexto são (TSAPATSIS, 2002):

a) Nanoposicionamento e nanocontrole de processos.

b) Mecanismos de nanoprecisão.

c) Descobrimento e/ou fabricação de superfícies super lisas.

d) Fabricação de estruturas em escalas nanométricas.

e) Análise das estruturas nessa escala.

f) Entendimento das propriedades físicas da matéria em escala nanométrica.

g) Por último, é essencial conseguir uma relação clara entre o mundo macroscópico e o nanoscópico.

Também foi realizada uma pesquisa para detectar o grau de desenvolvimento e de inovações tecnológicas sobre o tema. Para tanto foram utilizadas informações disponíveis no site do United States Patent and Trademark Office, onde se pode aferir a quantidade de depósitos de patentes americanas envolvendo especificações em nanômetros. O resultado desta pesquisa, conforme Figura 2, demonstra que o depósito de patentes americanas relacionadas à nanotecnologia também apresentou elevação nos últimos anos, embora de forma menos intensa se comparada com as publicações sobre o assunto.

Figura 2 - Número de depósitos de patentes x publicações acadêmicas em nanotecnologia

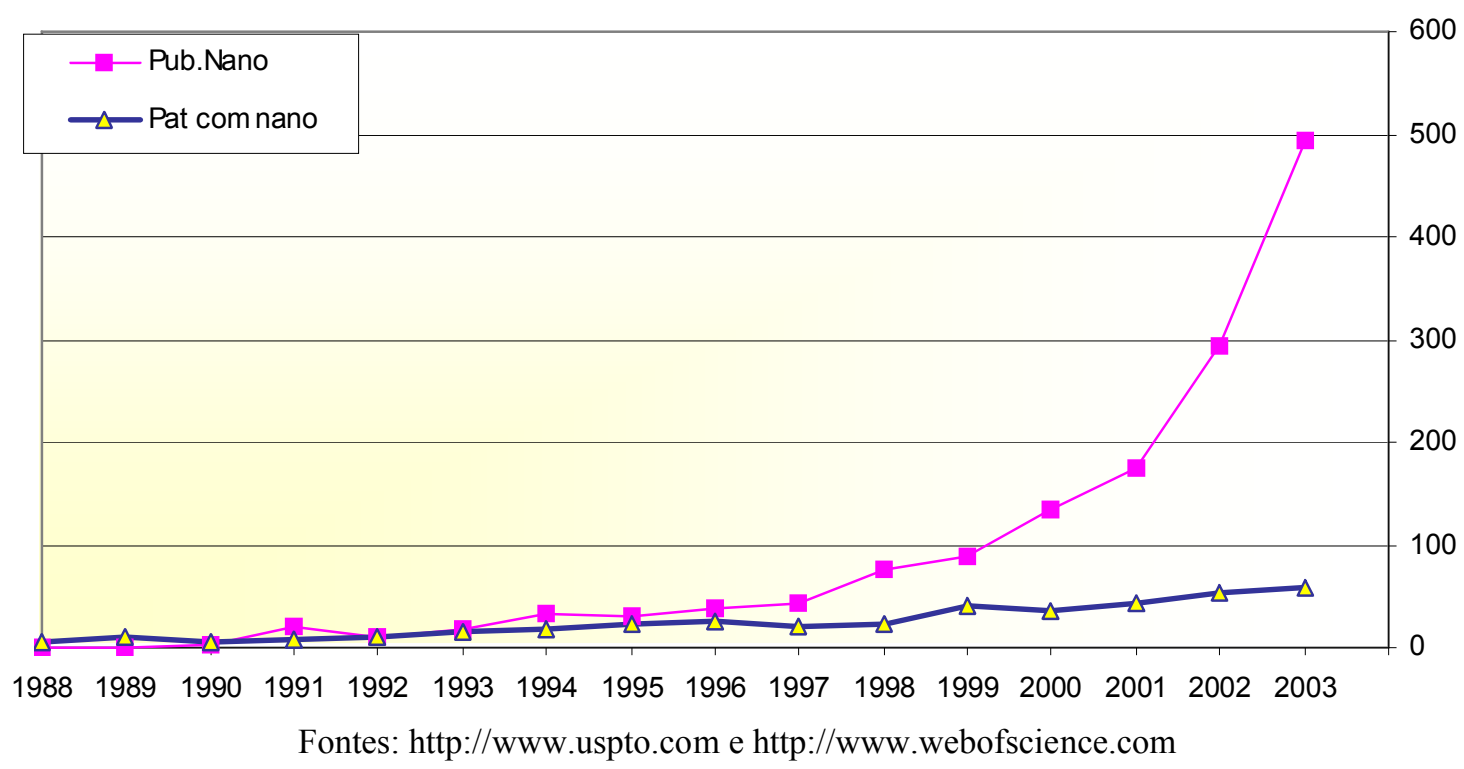

A escolha das patentes como documentos relevantes para a identificação de tendências tecnológicas deve-se, entre outras razões, ao fato de que são documentos objetivos, controlados por organismos oficiais, nacionais e internacionais, e pela necessidade e precaução de não se publicar 
informações sobre pesquisas e desenvolvimento tecnológico que possam vir a gerar patentes antes da devida proteção.

Houve, durante a realização da pesquisa, a preocupação com a pertinência entre comparações de dados e, por isso, todos os esforços neste sentido puderam ser atestados como factíveis diante da comparação das informações obtidas através de um gráfico de dispersão. Verifica-se uma associação linear entre os valores anuais de depósitos de patentes americanas e de publicações acadêmicas relacionadas a nanotecnologia, isto é, aos maiores valores de $\mathrm{x}$ correspondem os maiores valores de y. Esta comparação está representada na Figura 3.

De acordo com as informações obtidas, pode-se verificar que, em termos gerais, a nanotecnologia interessa tanto ao meio acadêmico quanto ao setor produtivo e isto pode ser explicado pelo vislumbre de um aproveitamento excepcional do conhecimento gerado em prol da utilização real e possível em diversos campos.

Figura 3 - Dispersão entre patentes depositadas e publicações

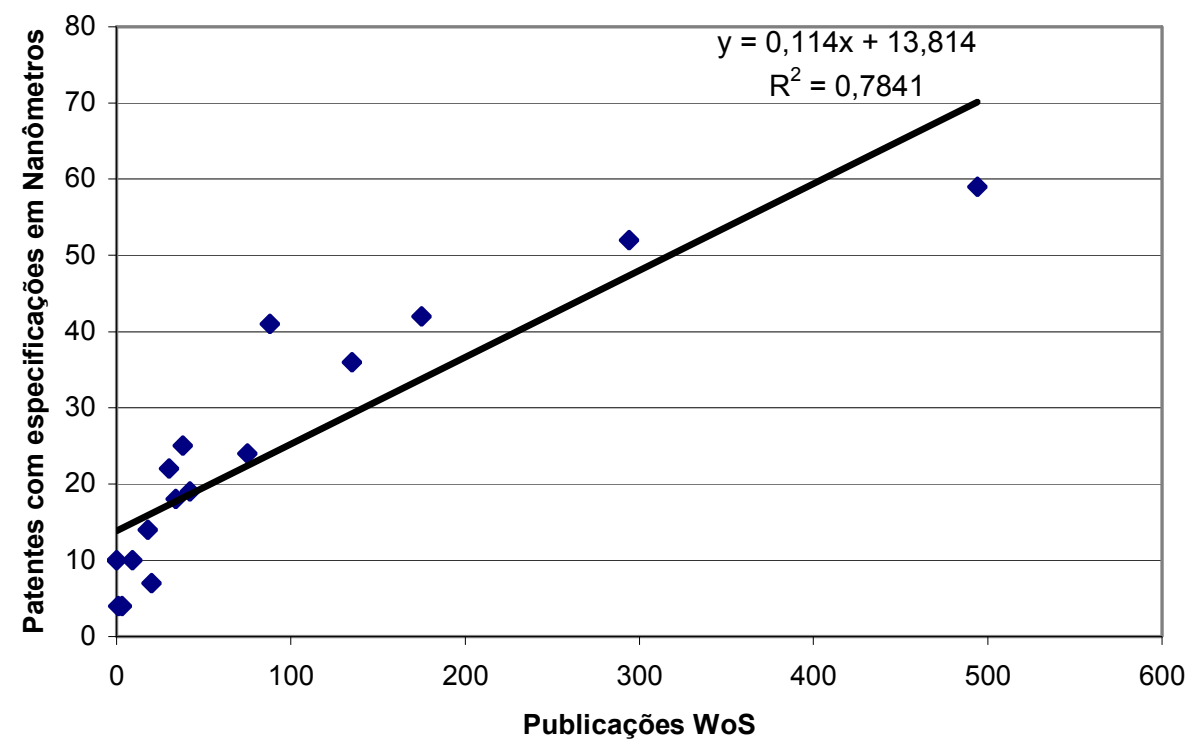

Fontes: http://www.uspto.com e http://www.webofscience.com

\section{Possibilidades de utilização e de novos mercados}

Por se tratar de área excepcionalmente nova e ainda pouco explorada, os possíveis campos de aplicação da nanotecnologia citados pela literatura devem ser considerados com certo grau de incerteza.

Conforme explicação anterior, a nanotecnologia cuida basicamente da manipulação de matéria em escala nanométrica. Logo, todas as matérias-primas formadoras de absolutamente todos os artefatos utilizados e produzidos pelo homem são propensos alvos de sua aplicação. 
Entretanto, de acordo com o relatório emitido no final década de 90 pela Interagency Working Group on Nanoscience, Engineering and Technology (ROCO, 1999), algumas das possíveis aplicações da nanotecnologia para a década atual são:

- Indústria automobilística e aeronáutica: materiais mais leves, pneus mais duráveis, plásticos não inflamáveis e mais baratos, etc. Os produtos finais irão variar de chapas de carroceria de automóveis que "se lembrarão" de sua forma anterior quando amassadas até compostos de aviões de peso "pluma" capazes de "sentir" rachaduras iminentes causadas por estresse e de realizar o auto-reparo (KOTLER \& SIMON, 2004)

- Indústria eletrônica e de comunicações: armazenamento de dados, telas planas, aumento na velocidade de processamento, etc.

- Indústria química e de materiais: catalisadores mais eficientes, ferramentas de corte mais duras, fluidos magnéticos inteligentes, etc.

- Indústria farmacêutica, biotecnológica e biomédica: novos medicamentos baseados em nanoestruturas, kits de auto-diagnóstico, materiais para regeneração de ossos e tecidos, etc.

- Setor de fabricação: novos microscópios e instrumentos de medida, ferramentas para manipular a matéria a nível atômico, bioestruturas, etc.

- Setor energético: novos tipos de bateria, fotossíntese artificial, economia de energia ao utilizar materiais mais leves e circuitos menores, etc.

- Meio-ambiente: membranas seletivas, para remover contaminantes ou sal da água, novas possibilidades de reciclagem, etc.

- Defesa: detetores de agentes químicos e orgânicos, circuitos eletrônicos mais eficientes, sistemas de observação miniaturizados, tecidos mais leves, etc.

Diante desse novo mercado aberto pelas possíveis aplicações da nanotecnologia, Roco (2003) alerta para a carência de profissionais na área nanotecnológica em um futuro não muito distante e discute várias parcerias entre universidades americanas e empresas do setor tecnológico a fim de garantir mão-de-obra especializada nos EUA.

\section{Investimentos e capital de risco em nanotecnologia e nanobiotecnologia}

Para se ter uma idéia do crescimento dos recursos governamentais vinculados a nanotecnologia, Defrancesco (2003) cita os investimentos globais em nanotecnologia por organizações governamentais que aumentaram de US\$ 432 milhões para quase US\$ 3 bilhões entre 1997 a 2003. E que estes fundos governamentais não vêm crescendo por causa da ciência em si, e sim, pela perspectiva que os políticos têm percebido desta nova área para a criação de empregos de 
forma similar ao que já aconteceu nos países centrais (EUA e Comunidade Européia) com a biotecnologia, a internet e as redes sem fio.

A NSF (National Science Foudation/USA) estima que existe atualmente cerca de 40.000 cientistas americanos com suas competências dedicadas à nanotecnologia. E que, para dar suporte a indústria da nanotecnologia - estimada em US\$ 1 trilhão em 2015, 800.000 trabalhadores americanos serão necessários (KOTLER \& SIMON, 2004).

De acordo com a NNI (National Nanotechnology Initiative/USA), entidade governamental criada em 2000 por Bill Clinton, a pesquisa em biotecnologia destina apenas $10 \%$ ou menos dos fundos governamentais globais para a nanotecnologia. Porém, o capital privado de risco (venture money) tem preferido ir na direção da nanobiotecnologia que em outros tipos de nanotecnologia (DEFRANCESCO, 2003).

A mesma posição do capital de risco internacional é indicada por Paull et al.(2003) e os números indicam que só o governo federal americano teve um crescimento de investimentos de US\$ 116 milhões para um valor orçado de US\$ 847 milhões em 2004. De 1999 a 2002, dos 900 milhões do capital privado de risco investido em nanotecnologia, 52\% foram para nanobiotecnologia conforme Figura 4.

Figura 4 - Aplicações dos fundos de capital de risco em nanotecnologia (1999 - 2002)

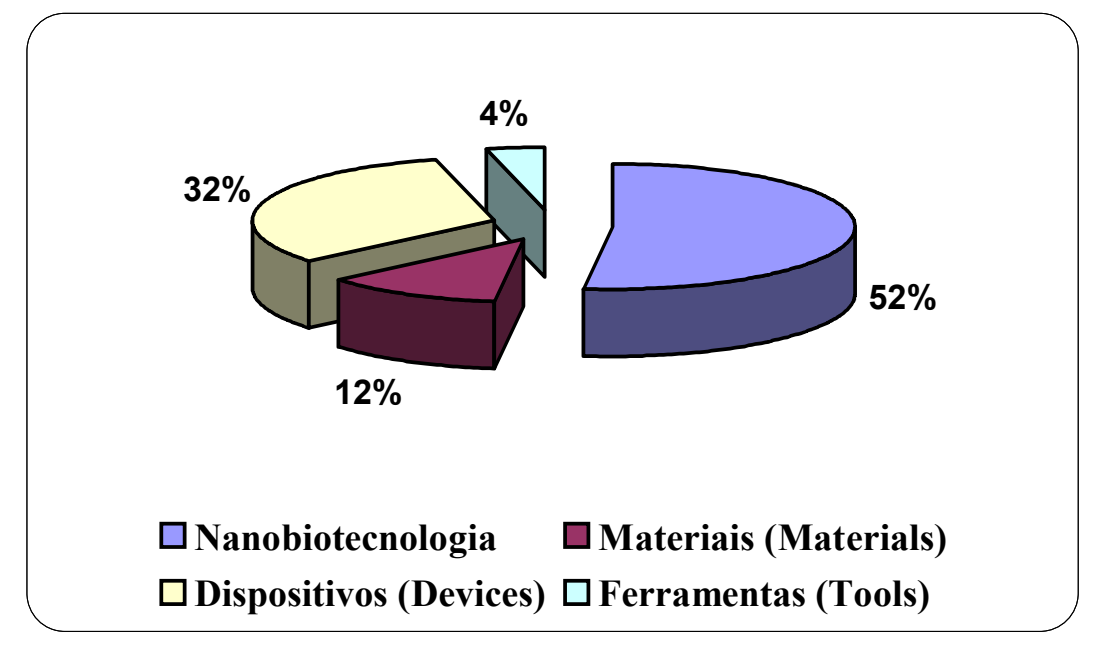

Fonte: Paull et al.(2003)

E, do montante investido em nanobiotecnologia, 54\% foram para descoberta de novas drogas e 37\% para realização de diagnósticos, como ilustrado na Figura 5.

No Brasil, através da Rede Brasileira de Nanotecnologia, criada pelo Ministério da Ciência e Tecnologia, foi encaminhada ao Congresso Nacional um Programa Plurianual para a Nanotecnologia (2004-2007) que está prevendo recursos de R\$ 77,7 milhões (VALLE, 2004). 
Silva Jr. (2004), em pequeno retrospecto histórico da nanociência e da nanotecnologia, menciona o lançamento do Programa Brasileiro de Nanociência e Nanotecnologia em 2001. Em 2002 o Ministério da Ciência e Tecnologia criou o Instituto do Milênio para Nanociências e, em 2003, era esperada a criação do Centro Nacional de Referência em Nanociência e Nanotecnologia.

Figura 5 - Distribuição do capital de risco na Nanobiotecnologia (1997 - 2002)

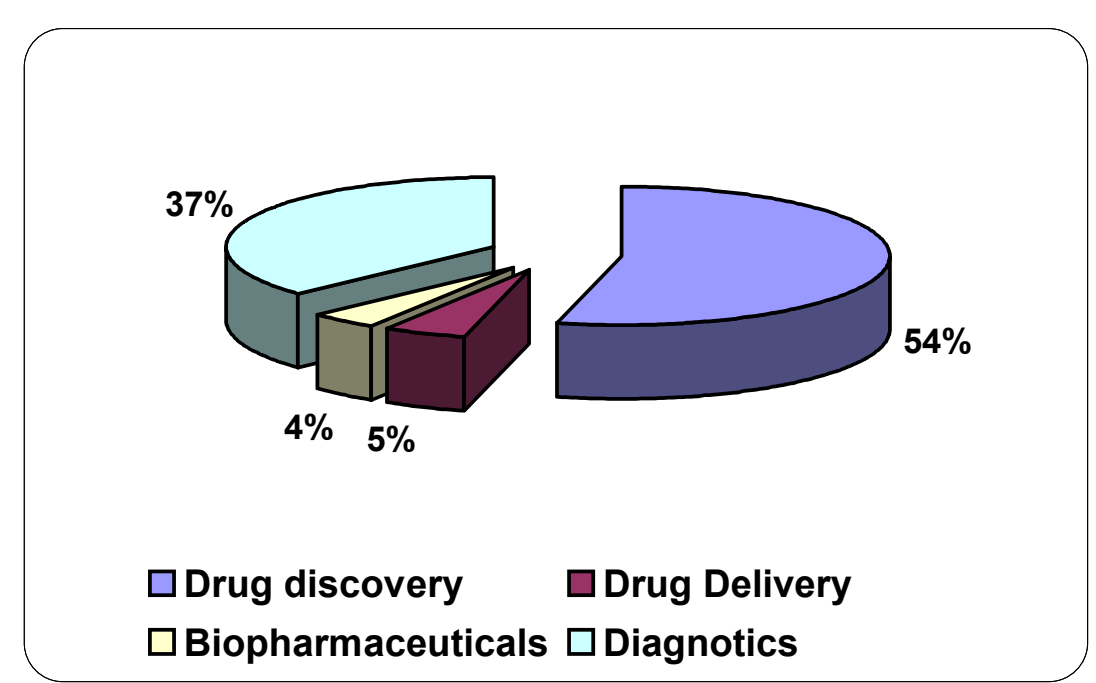

Fonte: Paull et al.(2003)

Das pesquisas em desenvolvimento no Brasil, podem ser citadas as do Instituto de Física da USP-SP que concentram esforços na produção de materiais nanoestruturados e dispositivos optoeletrônicos à base de nitreto de gálio $(\mathrm{GaN})$ e que poderão levar ao desenvolvimento da tecnologia nacional para a produção de nanodispositivos emissores de luz (LEDs e Lasers) na região do visível (verde e azul). Tais nanodispositivos têm aplicações importantes no armazenamento de dados (gravação e leitura de DVDs), painéis eletrônicos, iluminação de trânsito e etc.

\section{Conclusão}

A idéia básica da nanotecnologia já vem há bastante tempo ocupando a mente dos homens, entretanto, apenas recentemente o desenvolvimento tecnológico pode proporcionar a realização factual desta idéia. A possibilidade de alterar a composição das matérias em sua estrutura básica pode proporcionar ganhos com materiais potencialmente mais puros, leves, resistentes, baratos, precisos e adequados a basicamente todas as atividades desempenhadas pelo homem moderno.

A partir dos levantamentos realizados foi possível constatar significativo aumento de publicações científicas sobre o tema e também o aumento do número de patentes depositadas 
relacionadas à nanotecnologia. Esses indicadores demonstram interesse crescente por parte do meio acadêmico e do setor empresarial.

Como potenciais áreas de aplicação da nanotecnologia podem ser citadas as indústrias automobilística e aeronáutica, eletrônica e de comunicações, química e de materiais, farmacêutica, biotecnológica e biomédica, os setor de fabricação e energia, além de segurança e meio ambiente.

Quanto aos investimentos realizados, pode-se afirmar que o aporte global de recursos governamentais aplicados em nanotecnologia cresceu expressivamente nos últimos anos e que, em se tratando de capital de risco privado investido nessa área, a maior parcela dos investimentos vem sendo feita em nanobiotecnologia.

O Brasil ainda apresenta investimentos muito reduzidos em comparação aos países centrais - EUA e União Européia. Mesmo assim, torna-se relevante a criação de entidades nacionais que busquem o desenvolvimento deste setor que será um dos grandes propiciadores de empregabilidade e renda às futuras gerações.

\begin{abstract}
Nanotechnology means the ability to handle materials measured in nanometers in order to create structures with differentiated molecular organization. Nanobiotechnology refers to the interface between nanotechnology and biology aiming to create new materials and to develop new products and processes based on the growing capacity of modern technology to observe and manipulate atoms and molecules. This is an area of knowledge with vast potential to generate innovative process and products which start to raise economic and commercial possibilities and at the same time to exacerbate debates around ethical and environmental issues. This article intends to describe the state of the art and to identify future trends for nanotechnology research using date available on the Web of Science and United States Patent and Trademark Office together with survey of risk capital investments on nanobiotechnology and other nanotechnology.
\end{abstract}

Key-words: Nanotechnology; Nanobiotechnology; Innovation; Risk capital.

\title{
Referências
}

DEFRANCESCO, L. (2003) “Little science, big bucks”, Nature Publishing Group, v. 21, n. 10, pp.1127-1129

DREXLER, K.E. (1986) Engines of creation: the coming era of nanotechnology. Anchor Press/Doubleday, $1^{\text {a }}$. edição, New York

FEYNMAN, R.P. (1992) “There's plenty of room at the bottom”. Journal of Microelectromechanical Systems, v. 1, n. 1, pp.60-64

cross ${ }^{\text {ref }}$

KOTLER, P. \& SIMON, F. (2004) A construção de biomarcas globais: levando a biotecnologia ao mercado. Porto alegre: Bookman

KRUMMENACKER, M. \& LEWIS, J. (1995). Prospects in nanotechnology: toward molecular manufacturing. Hardcover, John Wiley \& Sons 
LÉVY, F. (1988). "Introduction à la nanotechnologie moléculaire". Texte de la présentation en Academie Interdisciplinaire des Sciences de Paris - AISP, Paris. Disponível em: <http://www.spirtech.com/flv/nano/>. Acessado em: 05 mai.2004.

PAUlL, R.; WOLFE, J.;HÉRBERT, P. \& SINKULA, M. (2003) “Investing in nanotechnology”, Nature Publishing Group, v. 21, n. 10, pp.1144-1147

ROCO, M.C. (2003) "Converging science and technology at the nanoscale: opportunities for education and training”, Nature Publishing Group, v. 21, n. 10, pp.1247-1249

ROCO, M.C.; WILLIANS, S. \& ALIVISATOS, P. (1999) "Nanotechnology research directions". IWGN workshop report: vision for nanotechnology research and development in the next decade. WTEC, Loyola College: Maryland. Disponível em http://www.ostp.gov/NSTC/html/iwgn/IWGN .research.directions/toc.htm. Acesso em 09 mai.2004.

SILVA JR, E.F. (2004). Rede para pesquisa em nanodispositivos semicondutores e materiais nanoestruturados. Disponível em: <http://www.comciencia .br>. Acesso em: 05 mai.2004.

SILVA, C. G. (2004). O que é nanotecnologia? Disponível em: <http://www.comciencia .br>._Acesso em: 05 mai.2004.

TSAPATSIS, M. (2002) "Molecular Sieves in the Nanotechnology Era”, AIChE Journal, v. 48, n. 4, pp. 654-655

\section{cross ${ }^{\text {ref }}$}

VALLE, S. (2004) "Da biotecnologia a nanobiosegurança”. Anais do Workshop em Nanotecnologia e Computação Inspirada na Biologia, n. 3, Rio de Janeiro.

\section{Dados completos do primeiro autor:}

Nome: Kleber Vieira Pina

Filiação institucional: Centro Federal de Educação Tecnológica Celso Suckow da Fonseca

Departamento: Diretoria de Pesquisa e Pós-Graduação / Programa de Pós-Graduação em Tecnologia

Função ou cargo ocupado: Mestrando

Endereço: Praia de Botafogo, 190 - 12 Andar, Botafogo, Rio de Janeiro/RJ, Brasil, CEP 22257900

Fone: (21)2559-5851

e-mail:kleber@fgv.br

Recebido para publicação em: 10/12/2005

Aceito para publicação em: 21/04/2006 